\title{
Neural Interconnectedness Between Thalamus, Cerebral Cortex and Cerebellum and its Pathophysiological Implications
}

\author{
Chan Kam Tim Michael* \\ Department of Dermatology, Hong Kong Academy of Medicine, China
}

Received: 眥: September 04, 2018; Published: 制 September 11, 2018

*Corresponding author: Dr. Chan Kam Tim Michael, Specialist in Dermatology, Hong Kong Academy of Medicine, Hong Kong SAR, China

\begin{abstract}
Advances in neurosciences, imaging techniques, magnetoencephalography and mathematical modelling enlightening us how our multi- billion neurons located in different parts of our brain integrate and interact synchronously in a concerted manner to enable us to exist not only biologically but in a conscious state of Mind process. Thalamocortical rhythm, resonance and cognitive, emotional and motor binding may give us the cue. This not only provide a basis for further understanding and research in neurocognitive science but may also provide new therapeutic insights into the management of previously difficult to treat neurological and psychiatric disorders. The cerebral cortex, thalamus and the primitive involuntary motor region like the olive and the cerebellum, albeit distantly located in the brain of Homo-sapiens has multiple, parallel excitatory and inhibitory interconnective neuronal synapses enabling an efficient integrated binding of motor, perceptual, cognitive and emotional function fundamental for our survival in a conscious state [1].
\end{abstract}

Abbreviations: T -DCS: Transcranial Direct Current Stimulation; R-TMS: Repetitive Transcranial Magnetic Stimulation; NMN: Mismatch Negativity; TCD: Thalamocortical Dysrhythmia; Cog-EM: Cognitive Emotional macrocircuit; MEG: Magnetoencephalography; VPL: Ventral Posterolateral Nuclei; VPM: Ventral Posteromedial Nuclei; MG: Medial Geniculate; LG: Lateral Geniculate; VL: Ventral Lateral; VAN: Ventral Anterior Nucleus

\section{Introduction}

The thalamus of our brain plays a pivotal role in this cognitivemotor-emotional binding neural circuitry. The thalamus is a complex anatomical structure found in all vertebrates in the animal world; with crucial physiological conscious and unconscious body activity. It is the center of control of much vital functions of our body like sleep, sensory information collection, dissemination, regulating autonomic nervous system and emotions. Acts as a conductor, it orchestrates signals in and out the cerebral cortex which reacts, plans and learns according through its various domains. Anatomically, the thalamus is well posited in the middle part of the mid brain as a paired structure at the dorsal end of the brain stem (Figure 1a) near the basal ganglion, hypothalamus, amygdala, nucleus accumbens and insula. Approximately with a size of a walnut; 3 to $4 \mathrm{~cm}$ in diameter; the thalamus consists of 30 to 40 nuclei. There are three basic types of thalamic nuclei; Relay, the Association and Nonspecific. The Relay nuclei receive specific definitive electrical signals and project this to the corresponding functional domains of distinct area in the cerebral cortex.

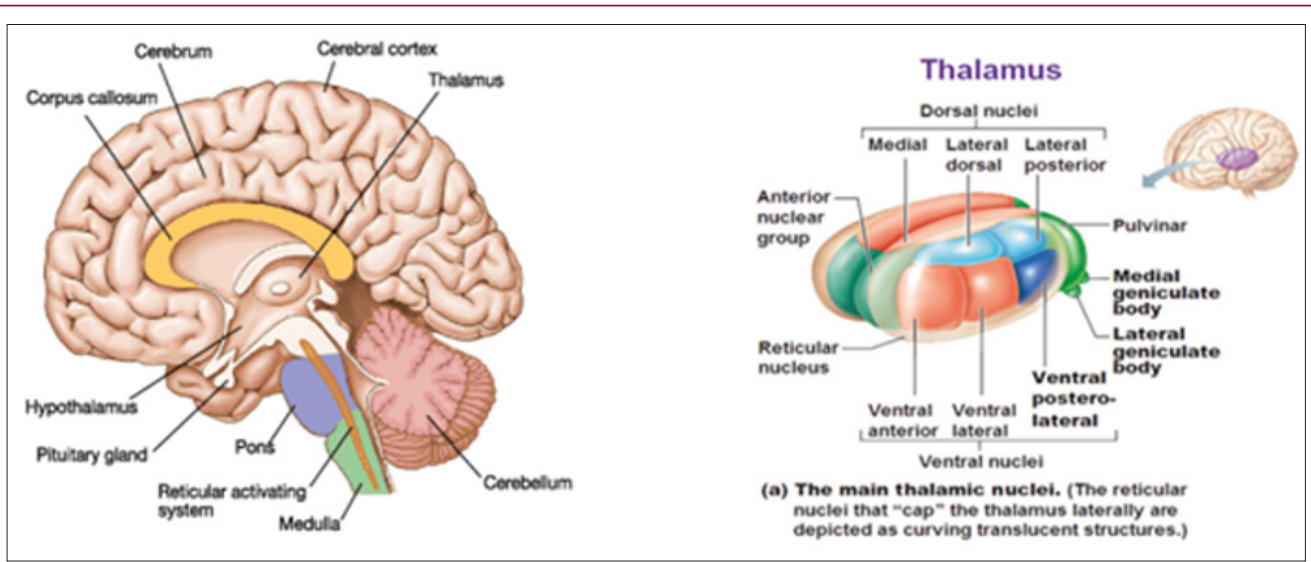

Figure 1: A: Source: Animalia World. B: It's a relay station for everything sensory that comes from the brain. 
The ventral posterolateral nuclei (VPL), ventral posteromedial nuclei (VPM), medial geniculate (MG) and lateral geniculate (LG) nuclei are thalamic nuclei which relay primary sensations to the cortex. The ventral lateral (VL) nuclei involved in feedback signal to the cerebellum while the ventral anterior nucleus (VAN) feedback basal ganglion output. Thus, a synaptic connective wiring networks are in place between the motor regions; cerebellum, basal ganglion and the conscious executor in the brain; the cerebral cortex. The Association nuclei receive signals input from the cerebral cortex which can be inhibitory and project back to the latter in cortex association areas so as to regulate activity. The last type called the nonspecific nuclei locates in the midline and intralaminar thalamus project widely throughout the cortex and may give a us the sense of alertness (Figure 1b) https://antranik.org/the-diencephalon/. Finally, there is the reticular nucleus which modulate neuronal signals going through the thalamus. The reticular nucleus encapsulated most of the thalamus and receives input from the cerebral cortex and dorsal thalamic nuclei.

Primary thalamic reticular nucleus efferent fibers project to dorsal thalamic nuclei only but does not project to the cerebral cortex. The thalamic reticular nucleus receives neuronal projections from the external segment of the Globus Pallidus. The Globus Pallidus is a component of basal ganglion of the brain which involved in the regulation of voluntary movement. Through disinhibition, neuronal signals were sent to the thalamus resulting in initiation of movement [2]. Thus, each nucleus of the thalamus is capable at rest in dispatching electrical signals and communications to specific anatomical domains to the corresponding cerebral cortex and areas of motor function. Through its association neurons, the signals are feedback to the thalamus and wired to the other structures like the amygdala and insula which in turn flow back to the cortex resulting in various perceptual sensations. Memory may be resulted if the wiring and signals involved the hippocampus. A feed forward and backward anatomical neuronal pathway is in place to enhance a secure balanced harmonious survival of the living creature during a hostile changing environment.

But how is this synaptic connection work in a conscious mind? With the advances of functional MRI imaging, brain wave detection by magnetoencephalography (MEG): a non-invasive investigation of human brain activity at high temporal resolution and mathematical modelling, neuroscientist now generally agree that the multimillion neurons in these areas of our brain are synchronously oscillating at a frequency depend on their states. High frequency oscillation in thalamocortical neurons was generated by dendritic calcium conductance [3]. Genetic predispositions, chemicals like neurotransmitters including psychotropic drugs, life events like trauma, brain injuries, malignancy, toxins and chronic inflammation are all possible contributing attributes affecting the firing rate frequency. This rhythmic oscillation as clinically referred by Llinás, et al. [1] as thalamocortical rhythm and mathematically predicted by Grossberg as Cognitive Emotional macrocircuit (Cog-EM) [1,4] (Figure 2) has been postulated in early years and proved valid to a significant extent of what might be happening.

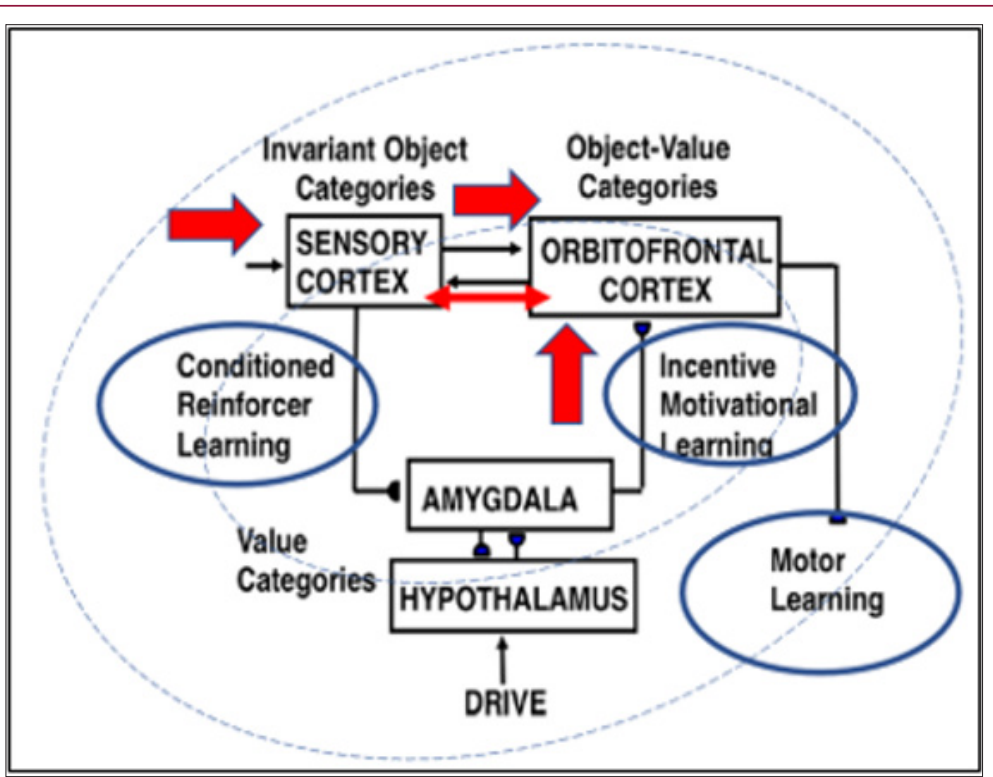

Figure 2: Source: Adapted from Grossberg (1971) and subsequent Cognitive-Emotional-Motor (CogEM) microcircuit (CogEM models show how invariant object categories in sensory cortex can activate value categories, also called drive representations, in the amygdala and hypothalamus, and object-value categories in the orbitofrontal cortex. Converging activation from an object category and its value category are needed to vigorously fire the corresponding object-value category. Achieving such convergence from the amygdala requires prior conditioned reinforcer learning and incentive motivational learning. When an object-value category fires, it can send positive feedback to its object representation and attentionally enhance it with valuederived activation. The motivationally enhanced object representation can then better compete with other object representations via a recurrent on center off-surround network and draw attention to itself. Closing the feedback loop between object, value, and object-value categories via a cognitive-emotional resonance can induce a conscious percept of having a emotion, or feeling, towards the attended object, as well as knowing what it is. As this resonance develops, the object-value category can generate output signals that can activate cognitive expectations and action commands through other brain circuits.) 
It predicts the cortex, thalamus, and the motor structure like the cerebellum oscillate and resonant in a harmonious low frequency conscious state exemplified by the beta or delta wave. The oscillating resonance bind these motor, perpetual, cognitive and emotional areas and functions in an alert conscious state. The representations of the external world in terms of perceived sensation and movement in relation to time will resonant and result in coherent synaptic oscillating activity allowing us to visualize, listen, awake and learn and plan and execute; i.e. thinking and movement in a state of consciousness. With this stable procure state of neuronal resonance, consciousness enables us to adapt more readily in addition to our primitive reflex second nature to our innate autonomic nervous system to an ever changing inside and outside environments. This translation of movement base motor learning neuronal activity to timely response into cognitive consciousness is undoubtedly an important and indispensable milestone to the development of the theory of Mind process and Self.

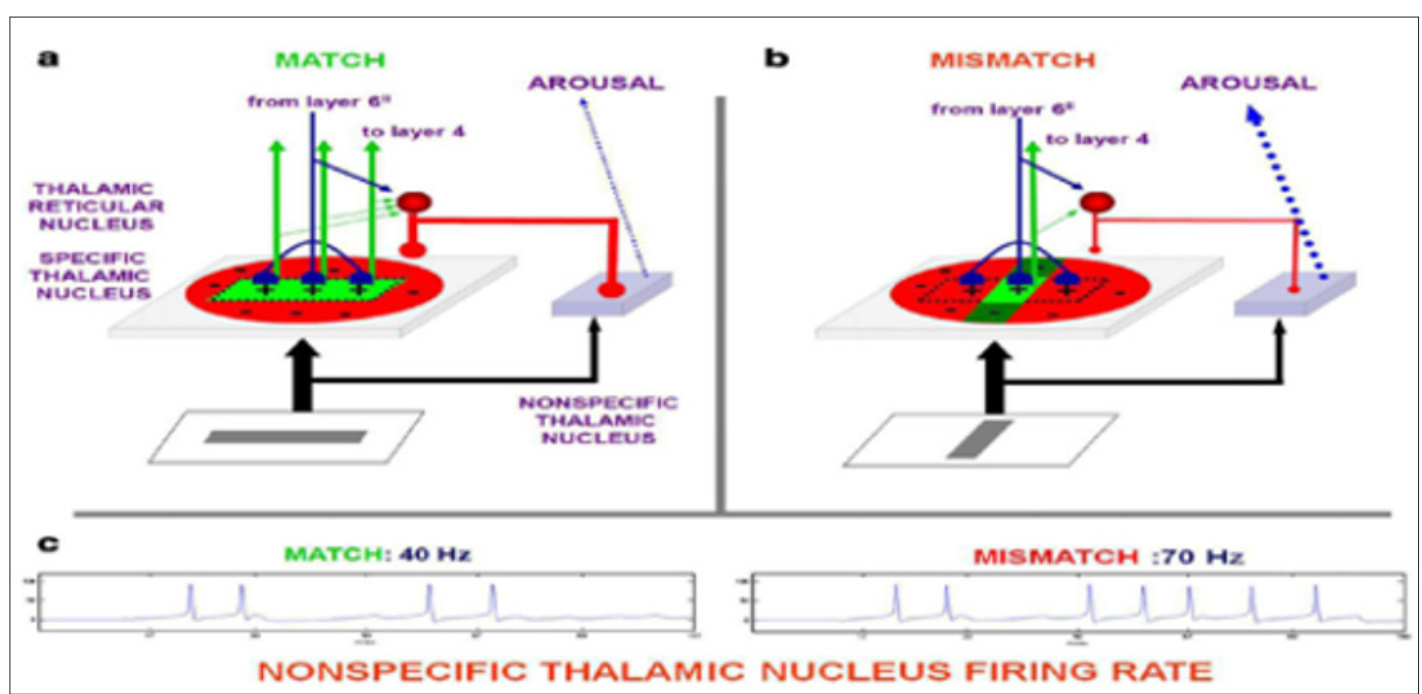

Figure 3: Nonspecific thalamic nucleus, cortical arousal regulation and mismatch negativity (NMN) (Grossberg 2008).

Sleep results when the thalamus enter a very low frequency oscillation referred as the gamma wave ( $40 \mathrm{~Hz}$ oscillations), this slow spurt and rippling enabling the cortex to follow and consciousness turn into a state of sleepiness of unconsciousness. Engel in his regarded publication coined the relationship between gamma wave and consciousness and hypothesize that synchronization of neuronal discharges can serve for the integration of distributed neurons into cell assemblies and that this process may underline the selection of perceptually and behaviorally relevant information [5]. This complete a day's natural conscious somatosensory motor activity and rest in deep sleep after dawn. However, if an abnormal rhythm is generated with very high or very low frequency, a so called thalamocortical dysrhythmia (TCD) or a Nonspecific thalamic nucleus, cortical arousal regulation and mismatch negativity (NMN) (Figure 3) may be resulted [6]. Neuroscientist now believed that this mismatch due to arousal or sustained attractor may produce an arena of neuropsychiatric symptoms and neurological brain diseases like tinnitus, schizophrenia, epilepsy, Parkinson's disease, depression, sleep insomnia, Tourette's syndrome, dystonia, autism, post traumatic brain syndrome, chronic neuropathic and phantom pain.

Based on the CogEM circuit model, if the there is an inputs imbalance with hypoactivity of orbitofrontal cortex or amygdala which provides motivationally directed goals, incentives or intentions drive improperly function, there will be a failure of the conscious state of resonance of the balanced neuro-circuits. Realistic memory representations and plans become fragmented and disparaged, schizophrenia with symptoms like hallucinations may be resulted due to failure in the mind processes [6]. Similarly, autistic sufferers may also fail to respond and apprehend other's actions with appropriate behavior due to aberrancy of this unwiring of thalamocortical circuits or CogEM mismatch. Nonetheless, not all behaviors of autism can be completely explained by the aforesaid [7]. Deep brain stimulation when applied precisely and accurately to the spot of the aberrant site of the cortex and thalamus can alleviate the symptoms as clinical evidences shown. However, if the correcting electrode is misplaced, hyper and hypo oscillating state may be resulted. Hallucinations, psychiatric co-morbidities and infections have been reported.

Transcranial direct current stimulation ( $\mathrm{t}$-DCS) and Repetitive transcranial magnetic stimulation (r-TMS) are newer modes of interfering the disequilibrium of resonant oscillations and has been documented in managing Parkinson's diseases, treatment resistant major depression and anxiety [8-10]. In sum, different parts of the brain are integrally connected through frequency oscillations in a resonant state. A bottom up input signals balanced by a top down volitions provide a match resonance conscious state giving us security with a sense of safety; nurturing, learning, development and motricity. A mismatch or asymmetry during vertical and horizontal integration of these neuronal signals will adversely affected the development of the living creature including human being especially during neonatal and adolescence period.

\section{References}

1. Llinás R, Ribary U, Contreras D, Pedroarena C (1998) The neuronal basis for consciousness. Philos Trans R Soc Lond B Biol Sci 353(1377): 18411849. 
2. Parent A, Hazrati LN (1995) Functional anatomy of the basal ganglia. I. The cortico-basal ganglia-thalamo-cortical loop. Brain Research Reviews 20(1): 91-127.

3. Pedroarena C, Llinás R (1997) Dendritic calcium conductances generate high-frequency oscillation in thalamocortical neurons. Proc Natl Acad Sci USA 94(2): 724-728.

4. Grossberg S (1971) On the dynamics of operant conditioning. Journal of Theoretical Biology 33(2): 225-255.

5. Engel AK, Fries P, Koenig P, Brecht M, Singer W (1999) Temporal binding binocular Rivalry, and consciousness. Consciousness and Cognition 8 (2): $128-151$.

6. Grossberg S, Bullock D, Dranias M (2008) Neural dynamics underlying impaired autonomic and conditioned responses following amygdala and orbitofrontal lesions. Behavioral neurosciences 122(5): 1100-1125.

\section{ISSN: 2574-1241}

DOI: $10.26717 / B J S T R .2018 .08 .001725$

Chan Kam Tim Michael. Biomed J Sci \& Tech Res

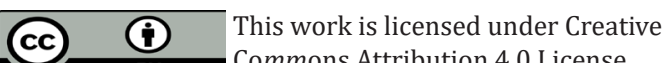

Submission Link: https://biomedres.us/submit-manuscript.php
7. Grossberg S (2017) Towards solving the hard problem of consciousness: The varieties of brain resonances and the conscious experiences that they support. Neural network 87: 38-95.

8. Elsner B, Kugler J, Pohl M, Mehrholz J (2016) Transcranial direct current stimulation (tDCS) for idiopathic Parkinson's disease. Cochrane Database Syst Rev.

9. Meron D, Hedger N, Garner M, Baldwin D (2015) Transcranial direct current stimulation (tDCS) in the treatment of depression: Systematic review and meta-analysis of efficacy and tolerability. Neuroscience \& Biobehavioral Reviews 57: 46-62.

10. Mi Z, Biswas K, Fairchild JK, Davis Karim A, Phibbs CS, et al. (2017) Trials. 18: 409. Repetitive transcranial magnetic stimulation (rTMS) for treatment-resistant major depression (TRMD) Veteran patients: study protocol for a randomized controlled trial. Trial 18(1): 409.

Assets of Publishing with us
BIOMEDICAL
RESEARCHES

